\title{
Cortical Auditory Evoked Potentials Reveal Changes in Audibility with Nonlinear Frequency Compression in Hearing Aids for Children: Clinical Implications
}

\author{
Teresa Y. C. Ching, Ph.D., 1 Vicky W. Zhang, Ph.D., 1 \\ Sanna Hou, M.Sc., 1 and Patricia Van Buynder, M.Sc. ${ }^{1}$
}

Hearing loss in children is detected soon after birth via newborn hearing screening. Procedures for early hearing assessment and hearing aid fitting are well established, but methods for evaluating the effectiveness of amplification for young children are limited. One promising approach to validating hearing aid fittings is to measure cortical auditory evoked potentials (CAEPs). This article provides first a brief overview of reports on the use of CAEPs for evaluation of hearing aids. Second, a study that measured CAEPs to evaluate nonlinear frequency compression (NLFC) in hearing aids for 27 children (between 6.1 and 16.8 years old) who have mild to severe hearing loss is reported. There was no significant difference in aided sensation level or the detection of CAEPs for $/ \mathrm{g} /$ between NLFC on and off conditions. The activation of NLFC was associated with a significant increase in aided sensation levels for $/ \mathrm{t} /$ and $/ \mathrm{s} /$. It also was associated with an increase in detection of CAEPs for $/ \mathrm{t} /$ and $/ \mathrm{s} /$. The findings support the use of CAEPs for checking audibility provided by hearing aids. Based on the current data, a clinical protocol for using CAEPs to validate audibility with amplification is presented.

KEYWORDS: Cortical auditory evoked potentials, nonlinear frequency compression, children, hearing aids, audibility

\footnotetext{
${ }^{1}$ National Acoustic Laboratories, Sydney, Australia. Address for correspondence: Teresa Y. C. Ching, Ph.D., National Acoustic Laboratories, Level 5, Australian Hearing Hub, 16 University Avenue, Macquarie University, NSW 2109 Australia (e-mail: Teresa.Ching@nal.gov.au).

The Use of Cortical Auditory Evoked Potentials in Diagnosis and Treatment of Hearing Disorders; Guest Editor, Bram Van Dun, Ph.D.
}

Semin Hear 2016;37:25-35. Copyright (C) 2016 by Thieme Medical Publishers, Inc., 333 Seventh Avenue, New York, NY 10001, USA. Tel: +1(212) 584-4662.

DOI: http://dx.doi.org/10.1055/s-0035-1570332.

ISSN 0734-0451. 
Learning Outcomes: As a result of this activity, the participant will be able to (1) discuss the use of cortical auditory evoked potentials (CAEPs) for validation of hearing aids; (2) describe how the use of nonlinear frequency compression in hearing aids affects audibility and the presence of CAEPs; and (3) describe how audibility and the presence of CAEPs relate to each other.

The implementation of universal newborn hearing screening has made it possible for infants born with hearing loss to be identified soon after birth. A challenge for audiologists is to provide them with auditory access to sounds by fitting hearing aids, verifying the fit to validated prescriptive procedures, and evaluating the effectiveness of amplification. Even though procedures for assessing hearing thresholds and fitting hearing aids incorporating individual real ear-to-coupler differences (RECDs) are well established (e.g., Seewald and Scollie ${ }^{1}$ ), methods for evaluating the effectiveness of amplification for young children are limited (see Bagatto et $\mathrm{al}^{2}$ for a review). For this reason, efforts have been directed into developing objective, electrophysiologic methods to complement subjective parental reports for clinical evaluation of hearing aids for infants. This article focuses on measuring cortical auditory evoked potentials (CAEPs) to speech sounds as an objective method for assessing audibility with amplification.

The CAEPs reflect the sum of synchronous, time-locked neural activity recorded at the scalp in response to an auditory stimulus. ${ }^{3}$ CAEPs can be evoked using auditory stimuli that are relatively long in duration and can be reliably recorded in infants and young children. ${ }^{4-6}$ In adults, the waveform of the evoked responses consists of a series of peaks or troughs (labeled P1, N1, P2, N2) that occur at $\sim 50$ to 250 milliseconds. In infants and young children, the evoked response is dominated by a large positivity (P1) at $\sim 100$ to 250 milliseconds followed by a late negativity at $\sim 250$ to 400 milliseconds. ${ }^{7}$ There has been extensive work on using P1 latency as a biomarker of auditory development. ${ }^{8-11}$ Other studies have used the presence of CAEPs to indicate that stimuli have been presented by a hearing device at levels sufficient to elicit neural activity in the auditory cortex, and hence must be audible. ${ }^{12-16}$ The absence of CAEPs, however, does not directly indicate that a sound is inaudible.
This is because individuals vary in the sensation level required for evoking cortical activity of sufficient strength for it to be detected with current methods (e.g., Glista et $\mathrm{al}^{17}$ and Van Dun et $\mathrm{al}^{18}$ ). CAEPs can be evoked by tonal and speech stimuli. For hearing aid evaluation, speech stimuli have higher face validity and are available in clinical systems for measuring auditory evoked potentials.

In this article, we will first provide a brief overview of evidence on the use of cortical measurements for hearing aid evaluation. Second, we will describe an experiment that used measurements of CAEPs to evaluate whether the use of nonlinear frequency compression (NLFC) in hearing aids improved children's access to speech sounds. Finally, we propose a protocol that enables clinicians to evaluate the effectiveness of hearing aids for young children in a clinical setting.

\section{EVIDENCE ON THE USE OF CORTICAL AUDITORY EVOKED POTENTIALS FOR HEARING AID EVALUATION}

For CAEPs to be used for validation of hearing aid fitting, ${ }^{13}$ the relationship between audibility and presence of CAEPs needs to be established. Van Dun et al showed that greater audibility was significantly correlated with greater certainty that CAEPs were present for infants with sensory/neural hearing loss (SNHL) in either aided or unaided conditions, although audibility accounted for only $9 \%$ of variance in probability levels. ${ }^{18}$ In a similar vein, Gardner-Berry et al (this issue) found a significant relationship between estimated audibility of stimuli and presence of CAEPs for infants below 3 years of age, both for children with or without SNHL and children with auditory neuropathy spectrum disorder (ANSD). ${ }^{19}$ These findings lend support for the use of CAEPs for assessing audibility with hearing aids, especially for people who are unable to provide reliable 
behavioral responses or in cases where there is uncertainty over hearing thresholds, such as those with ANSD.

Further support is provided by other studies that examined the relationship between CAEPs and functional outcomes for aided infants and children. Golding et al investigated the relationship between aided CAEPs and real-life functioning in 28 infants with either SNHL or ANSD. ${ }^{20}$ Functional performance was measured using the Parents' Evaluation of Children's Aural/Oral Performance (PEACH) scale. ${ }^{21}$ On average, higher detection rates of CAEPs were associated with higher PEACH scores. A recent study by Gardner-Berry et al on 12 infants with ANSD showed that the presence of more evoked responses to speech stimuli was associated with higher PEACH scores. ${ }^{22}$ In school-aged children with ANSD, Rance et al showed that the presence of aided CAEPs was associated with better speech perception ability. ${ }^{23}$ These studies suggest that children for whom CAEPs were detected for a greater proportion of sound stimuli presented also used their aided hearing ability more effectively in real life.

There is growing interest in using speechevoked CAEPs to objectively determine whether a child with hearing loss detects speech sounds at conversational levels and processes them at the level of the auditory cortex. This approach may be valuable for selecting signalprocessing features in hearing aids that can be potentially beneficial for young children, because it is crucial that the impact of these features on audibility of speech be evaluated. ${ }^{24}$ The frequency-lowering feature, for example, has been designed to address the difficulty of people with hearing impairment to perceive high-frequency sounds by presenting high frequencies at a lower frequency region. ${ }^{25}$ One method of frequency-lowering, NLFC, has been implemented in commercial hearing aids for children. The processing affects only frequencies above a preset "cutoff frequency", leaving the lower frequencies unaltered. Above the cutoff frequency, frequency components in the incoming signal are compressed by a progressively increasing amount before they are delivered to the output. The amount of compression is determined by a frequency-compres- sion ratio. The NLFC causes a wide range of frequencies above the cutoff frequency to be presented to a narrower range of frequencies at the output of the hearing aid, so that highfrequency components of speech can become audible at a lower-frequency region. For children with hearing loss, access to speech sounds that span the speech frequency spectrum with their hearing aids underpins development of auditory/oral communication skills. ${ }^{26-28}$

Two recent studies reported the use of aided CAEPs to assess the effect of NLFC for children. In a pilot study, Glista et al compared aided CAEPs to estimated sensation levels of auditory stimuli for five children with hearing impairment in two aided conditions (NLFC on and NLFC off). ${ }^{17}$ The stimuli were tone bursts at $2 \mathrm{kHz}$ and $4 \mathrm{kHz}$ presented via direct audio input to hearing aids worn in the better ear. For the $2-\mathrm{kHz}$ tone burst, CAEPs were detected in both aided conditions. For the 4-kHz tone burst, CAEPs were present for only one child when NLFC was deactivated, but for all five children when NLFC was activated. Although based on a very small sample, the findings suggest that measurement of CAEPs may be sensitive to the effects of NLFC and that the processing may have augmented audibility of high-frequency tone bursts for individual listeners. A recent study by Zhang et al reported aided CAEPs evoked using short speech sounds in 39 children with hearing impairment. ${ }^{29}$ The stimuli were $/ \mathrm{g} /, \mathrm{t} / \mathrm{t} / \mathrm{s} /$ presented at 55- and $65-\mathrm{dB}$ sound pressure level (dB SPL) in the sound field. The study found a significant increase in the detection rate of CAEPs for /s/ at 55-dB SPL when children used new NLFC hearing aids than when they used their own hearing aids with conventional processing. As the audibility of the speech stimuli amplified via the different hearing aids was not quantified, it remained uncertain as to whether the difference in detection rate between the two aided conditions was related to variations in high-frequency audibility due to activation of NLFC or to other differences between the two hearing aid settings that were unrelated to NLFC. Nevertheless, these preliminary studies suggest that there is much potential for using CAEPs to assess aided audibility in children, but research is needed 
to increase understanding of the relationship between the detection rate of aided CAEPs and sensation levels of speech stimuli with NLFC activation.

\section{CURRENT RESEARCH}

The purpose of this study was to determine (1) how NLFC affects audibility; (2) how NLFC affects the presence of CAEPs; and (3) how do audibility and the presence of CAEPs relate to each other.

\section{MATERIALS AND METHODS}

Participants included 27 children with sensory/ neural hearing loss (mean $=11.6$ years; range: 6.1 to 16.8 years) recruited as part of a multisite study designed to examine the effectiveness of NLFC for children. For that study, data on speech perception, speech production, and functional performance were gathered in a crossover controlled trial of NLFC with extended periods of familiarization. Participants in this report consisted of children in that study who consented to measurement of CAEPs. The study protocol was approved by an institutional ethics review board.

The participants' audiograms are shown in Fig. 1. All children are experienced users of hearing aids with conventional processing.
Once enrolled in the study, new NLFC hearing aids were fit according to the standard national protocols of Australian Hearing to match NAL-NL1 targets while incorporating RECDs in personal fittings. ${ }^{30-33}$ Individually measured or age-appropriate average RECDs were used in deriving prescriptive targets, and hearing aids were measured and adjusted in an HA2-2cc (2 cc coupler for HA2) coupler to match targets at low $(50 \mathrm{~dB})$, average $(65 \mathrm{~dB})$, and high $(80 \mathrm{~dB})$ inputs and maximum power output as closely as possible. The NLFC settings (i.e., cutoff frequency and frequency compression ratio) were adjusted away from the manufacturer's default settings for individual audiograms in the direction of providing greater audible bandwidth for 25 ears. Adjustments in the direction of providing less audibility were performed for three ears, based on subjective feedback about sound quality.

\section{Measurement of Aided Cortical Auditory Evoked Potentials}

CAEPs were recorded by using the HEARLab system (Frye Electronics, Tigard, OR). The test stimuli were $/ \mathrm{g} /, / \mathrm{t} /$, and $/ \mathrm{s} /$, with durations of 21, 30, and 100 milliseconds, respectively (see Fig. 2). In a sound-treated room, the stimuli were presented from a loudspeaker positioned at 0 degrees azimuth at a distance
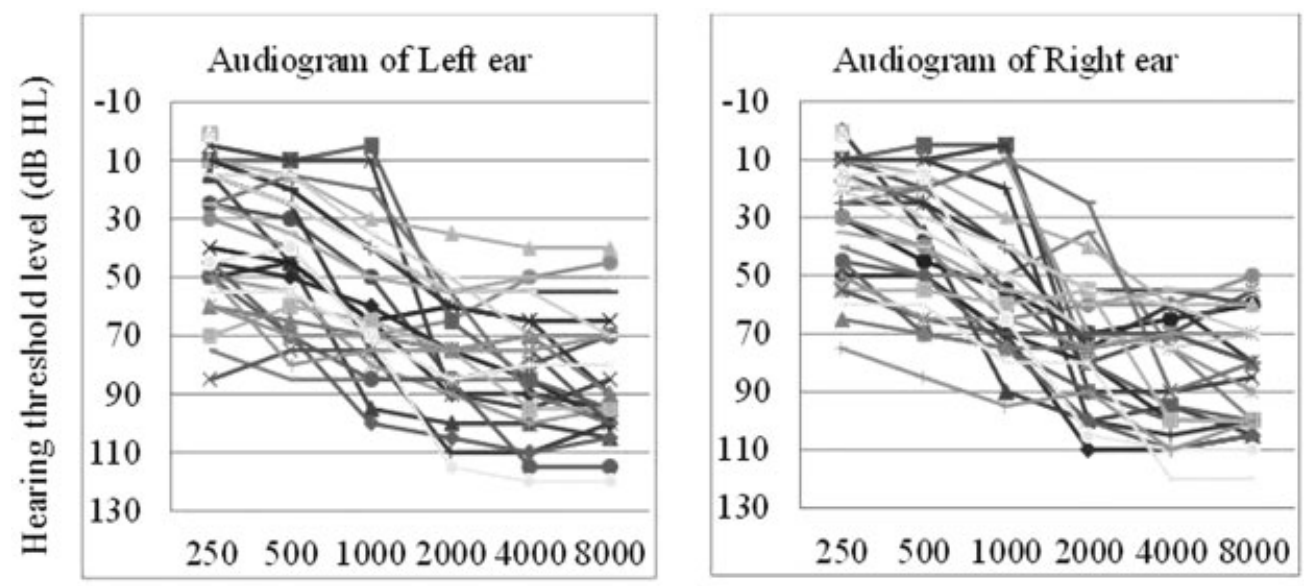

Frequency $(\mathrm{Hz})$

Figure 1 Audiograms of participants. Abbreviation: HL, hearing loss. 


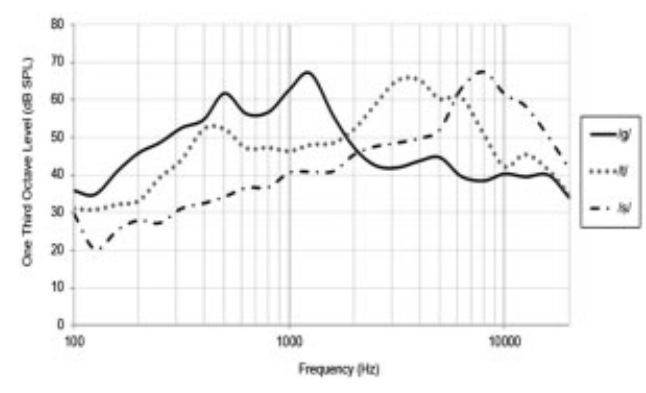

Figure 2 Spectra for the speech stimuli used for measuring cortical auditory evoked potential, with overall levels normalized to $65 \mathrm{~dB}$ SPL.

of $1 \mathrm{~m}$ from the subject position. The overall presentation levels were 55- and 65-dB SPL in the sound field. The participant was seated in a comfortable chair watching a video with the sound turned off, wearing hearing aids at their personal settings. Three electrodes were used for acquisition: the active electrode was placed at the vertex $(\mathrm{Cz})$, the reference electrode on the mastoid (M1), and the ground electrode on the forehead $(\mathrm{Fz})$. During recording, an automated detection algorithm in the HEARLab ${ }^{\mathrm{TM}}$ system analyzed the electroencephalogram to generate a significance level ( $p$ value), based on at least 100 accepted epochs (range: 100 to 224) for each stimulus. CAEPs were deemed to be present if the $p$ value was $<0.05$.

Aided CAEPs were measured with the children wearing their personal hearing aids and the new hearing aids in two conditions: NLFC activated and deactivated. The measurements were completed on separate test sessions, after each participant had a familiarization period with each of the aided conditions for 4 to 8 weeks. The order of test condition was counterbalanced across participants.

\section{Calculation of Audibility}

The audibility of speech stimuli was calculated by adding the stimulus level to the real earaided gain and then compared with the sum of the hearing threshold (in decibels of HL) converted to its equivalent SPL in the ear canal. The hearing aids were measured in an HA2-cc coupler at low- and average-level inputs. The coupler gain was added to the individual's RECDs to give real ear-aided gain. The spec- tral characteristics of each stimulus were measured in one-third octave bands in $\mathrm{dB}$ SPL in the free field. These stimulus levels were added to the real ear-aided gain to give aided stimulus level in the ear canal. For NLFC deactivated, the aided sensation level of a stimulus at each one-third octave band was the difference between the aided level of the stimulus and the audiometric hearing threshold interpolated in that band, expressed as $\mathrm{dB}$ SPL in the ear canal. For NLFC activated, the input frequencies that were presented at certain output frequencies when specific frequency compression thresholds and ratios were used for each fitting were determined by using the hearing aid fitting software. Measurements of the hearing aids in an HA2-2cc coupler confirmed the validity of the method. The aided sensation level of the stimulus was then estimated by comparing the aided stimulus level to the hearing thresholds. Audibility of each stimulus was quantified as the maximum aided sensation level across onethird octave bands in the better ear.

\section{RESULTS}

To address the first research question regarding how NLFC affects audibility, the aided sensation levels of $/ \mathrm{g} /, / \mathrm{t} /, / \mathrm{s} /$ for two NLFC conditions were examined (see Fig. 3). Analyses of variance with aided sensation level as dependent variable, processing (NLFC on versus off), presentation level $(55,65)$ and stimuli $(/ \mathrm{g} / / \mathrm{t} /$ $/ \mathrm{s} /$ ) as categorical variables indicated that the

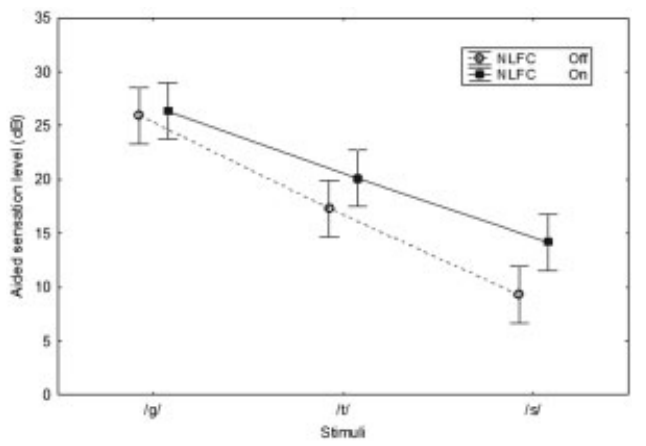

Figure 3 Mean aided sensation levels of stimuli when nonlinear frequency compression (NLFC) was activated (filled symbols) and deactivated (open symbols). Vertical bars denote $95 \%$ confidence intervals. 
main effect of presentation level was significant $(F[1,26]=806.39, p<0.0001)$. The main effect of NLFC was significant $(F[1$, $26]=7.91, p=0.009)$, and the main effect of stimuli was significant $(F[2,52]=74.0$, $p<0.0001)$. There was significant interaction effects between NLFC and stimuli ( $F[2$, $52]=5.39, p=0.007)$. Post hoc analysis using the Tukey's honest significant difference test indicated that on average, sensation levels were higher when NLFC was activated than when it was deactivated, for $/ \mathrm{t} /(p=0.047)$ and $/ \mathrm{s} /$ $(p<0.001)$.

To address the second question on how NLFC affects the presence of CAEPs, the detection rates for NLFC on versus off were compared. Table 1 shows the detection rates of CAEPs, calculated as a ratio of number of detection versus number of stimuli presented, expressed as a percentage. A $z$ test of difference between proportions indicated that on average, the detection rate of CAEPs for $/ \mathrm{t} /$ was significantly higher when NLFC was activated than when it was deactivated. There was a similar trend for $/ \mathrm{s} /$, although the difference did not reach the 5\% significance level.

Fig. 4 shows $p$ values of the CAEPs measured in the NLFC-activated condition versus $p$ values in the deactivated condition, separately for $/ \mathrm{g} /, \mathrm{t} / \mathrm{t}$, and $/ \mathrm{s} /$. In each panel, the data points in the lower right-hand quadrant depict measurements for CAEPs that were absent when NLFC was deactivated but present $(p<0.05)$ when NLFC was activated.

To address the third question on how audibility and the presence of CAEPs relate to each other, product moment correlation analysis was performed between estimated sensation levels and $p$ values (log-transformed) for all 452 recordings of CAEPs (including own aid condition, new hearing aids with NLFC on, and NLFC off conditions). On average, there was a significant negative correlation $(r=$ $-0.17, p<0.001)$, suggesting that higher sensation levels were associated with lower $p$ values. At positive sensation levels $(>0 \mathrm{~dB})$, the detection rates were 93,90 , and $76 \%$ for $/ \mathrm{g} /, / \mathrm{t} /$, and /s/, respectively. At sensation levels greater than $10 \mathrm{~dB}$, the detection rates were 96,90 , and $77 \%$ for $/ \mathrm{g} /, / \mathrm{t} /$, and $/ \mathrm{s} /$, respectively. Table 2 summarizes the detection rate of each stimulus for narrow ranges of aided sensation levels for each stimulus.

To investigate whether the presence of CAEPs were related to the degree of hearing loss, multiple regression analysis was performed with $p$ values (log-transformed) as dependent variable and hearing thresholds at $2 \mathrm{kHz}$ and $4 \mathrm{kHz}$ as independent variables. The analysis showed a weak but significant relationship $(F=[2,449]=16.85, p<0.0001)$, accounting for $7 \%$ of variance. Hearing thresholds at $4 \mathrm{kHz}$ contributed significantly to predicting $p$ values of CAEPs (beta $=0.24, p<0.0001$ ). When only CAEPs measured with conventional hearing aid processing (own hearing aids, new hearing aids with NLFC deactivated), the detection rate of CAEPs for $/ \mathrm{s} /$ was $74 \%$ (48 of 74 recordings) and for / $\mathrm{t} /$ was $87 \%$ (58 of 67 recordings) for hearing thresholds at $4 \mathrm{kHz}$ better than $90 \mathrm{~dB}$ HL. The corresponding detection rate for $/ \mathrm{s} /$ was reduced to $55 \%$ (16 of 29 recordings) and for $/ \mathrm{t} /$ to $81 \%$ (25 of 31 recordings) for more severe hearing loss. When only CAEPs measured with NLFC activated were considered, the detection rate for $/ \mathrm{s} /$ was $81 \%$ (29 of 36 measures) and for $/ \mathrm{t} /$ was $97 \%$ (35 of 36 measures) for hearing thresholds at $4 \mathrm{kHz}$

Table 1 Detection Rates of CAEPs

\begin{tabular}{|c|c|c|c|c|c|c|c|}
\hline \multirow[t]{2}{*}{ Stimulus } & \multicolumn{3}{|l|}{ NLFC off } & \multicolumn{3}{|l|}{ NLFC on } & \multirow{2}{*}{$\begin{array}{l}\text { Difference } \\
p \text { Value }\end{array}$} \\
\hline & No. Detected & No. Presented & $\%$ Detection & No. Detected & No. Presented & $\%$ Detection & \\
\hline /g/ & 46 & 48 & 95.8 & 46 & 47 & 97.9 & 0.58 \\
\hline$/ t$ & 39 & 47 & 83.0 & 46 & 47 & 97.9 & $0.01^{*}$ \\
\hline /s/ & 32 & 45 & 71.1 & 39 & 48 & 81.3 & 0.25 \\
\hline
\end{tabular}

Abbreviations: CAEP, cortical auditory evoked potential; NLFC, nonlinear frequency compression.

Note: Results are expressed as a percentage for two conditions of nonlinear frequency compression (NLFC off and NLFC on).

*Difference in proportion between conditions that is significant at $p<0.05$. 

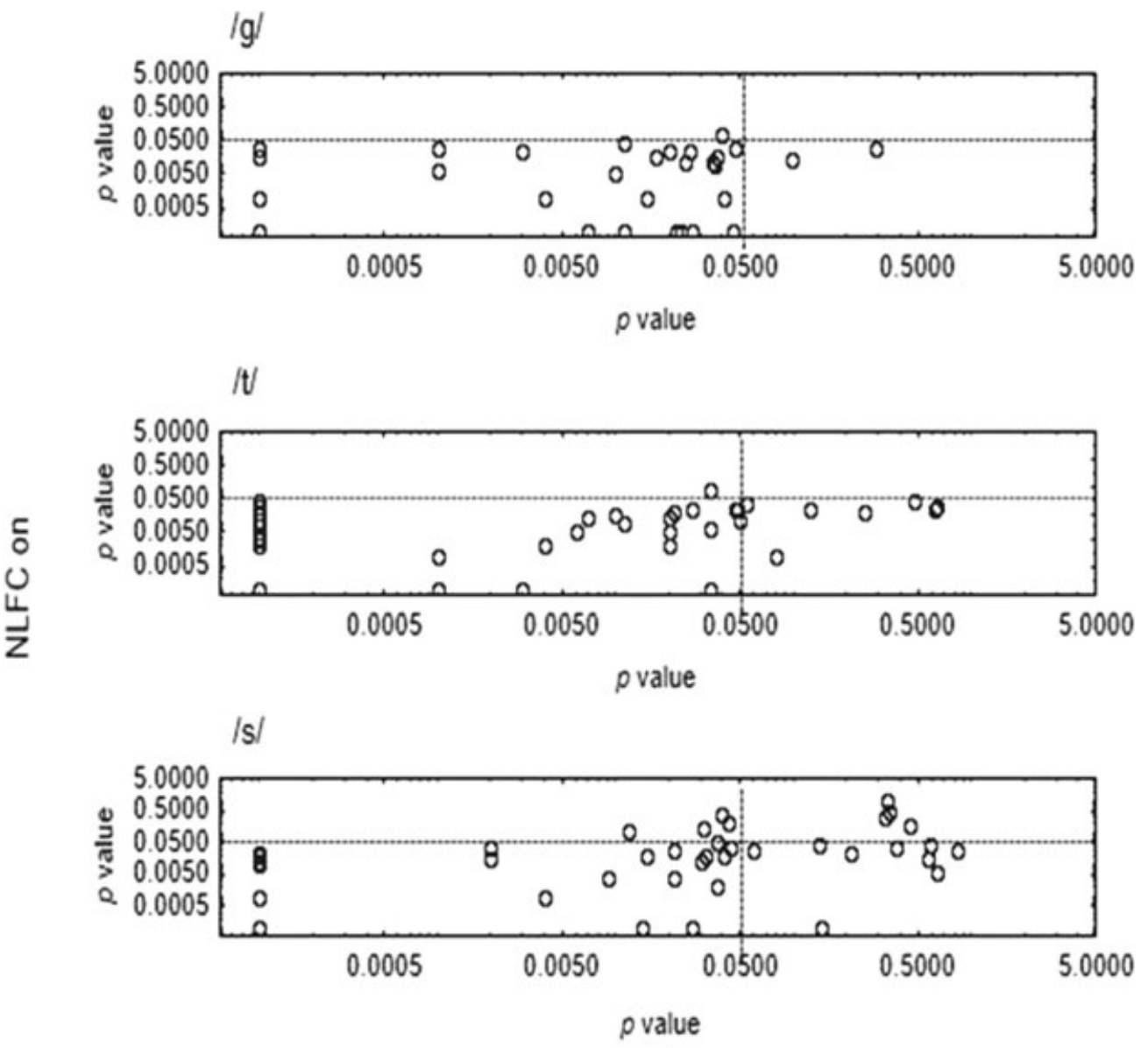

\section{NLFC off}

Figure 4 Probability level ( $p$ value) of measurements of cortical auditory evoked potentials when nonlinear frequency compression (NLFC) was on (y-axis) versus $p$ value when NLFC was off ( $x$-axis), separately for each stimulus (/g/, /t/, /s/ from top to bottom panels). In each panel, data points in the bottom left quadrant depict measurements that were significant $(p<0.05)$ in both NLFC conditions. Those in the top left quadrant depict measures that were significant when NLFC was off, but not when it was on. The top right quadrant shows measurements that were not significant irrespective of whether NLFC was activated. The bottom right quadrant depicts measurements that were significant when NLFC was on that were not significant when it was off.

better than $90 \mathrm{~dB}$ HL. The corresponding rates were $78 \%$ (14 out of 18 measures) for $/ \mathrm{s} /$ and $100 \%$ for $/ \mathrm{t} /$ (17 measures) for more severe hearing loss.

\section{DISCUSSION}

The findings in this study show that CAEPs for speech stimuli were present for most stimuli with most participants. The detection rates of CAEPs at positive sensation levels were higher than those reported in previous studies on young children with hearing loss (e.g., Van Dun et al, ${ }^{18}$ Chang et al, ${ }^{34}$ Gardner-Berry et $\mathrm{al}^{19}$ ). In those studies, CAEPs were present for $68 \%$ or 71 to $78 \%$ for $/ \mathrm{m} /, / \mathrm{t} /, / \mathrm{g} /$ presented at positive sensation levels. ${ }^{18,34}$ These lower rates may relate to factors including the age of participants, hearing loss configuration, and hearing aid settings. Previous studies included infants under 3 years of age assessed in either aided or unaided conditions, whose auditory 
Table 2 Detection Rates of CAEPs

\begin{tabular}{|c|c|c|c|c|c|}
\hline & $\begin{array}{l}\text { Sensation } \\
\text { Level (dB) }\end{array}$ & $\begin{array}{l}\text { No. of } \\
\text { Participants }\end{array}$ & $\begin{array}{l}\text { No. of } \\
\text { Detections }(p<0.05)\end{array}$ & $\begin{array}{l}\text { No. of Stimuli } \\
\text { Presented }\end{array}$ & $\begin{array}{l}\% \\
\text { Detection }\end{array}$ \\
\hline \multirow[t]{4}{*}{ /g/ } & $<0$ & 0 & - & - & - \\
\hline & $0-9$ & 7 & 4 & 7 & - \\
\hline & 10-19 & 16 & 43 & 47 & 95.9 \\
\hline & $\geq 20$ & 32 & 95 & 99 & 96.0 \\
\hline \multirow[t]{4}{*}{$/ \mathrm{t} /$} & $<0$ & 5 & 4 & 7 & - \\
\hline & $0-9$ & 13 & 19 & 22 & 86.4 \\
\hline & $10-19$ & 18 & 45 & 49 & 91.8 \\
\hline & $\geq 20$ & 28 & 65 & 73 & 89.0 \\
\hline \multirow[t]{4}{*}{ /s/ } & $<0$ & 12 & 10 & 25 & 40.0 \\
\hline & $0-9$ & 18 & 30 & 40 & 75.0 \\
\hline & 10-19 & 20 & 35 & 44 & 79.5 \\
\hline & $\geq 20$ & 22 & 29 & 39 & 74.4 \\
\hline
\end{tabular}

Abbreviation: CAEP, cortical auditory evoked potential.

Note: Results are expressed as a percentage for different ranges of stimulus sensation level. Detection rates for stimuli numbers that were less than 10 were not shown.

experience with speech sounds were limited, and for whom there were considerable uncertainties about hearing thresholds that were used for estimating sensation levels. The uncertainty of threshold estimates and the potential for thresholds to have changed over time between cortical measurement and behavioral audiometry are likely to have contributed to missing cortical responses for stimuli estimated to be above hearing thresholds or responses occurring for stimuli estimated to be below hearing thresholds. The present study included children at school age who used spoken language as the primary mode of communication and for whom reliable behavioral thresholds were established. Also, they were longtime users of hearing aids and had extended familiarization periods with the hearing aid settings that were well matched to prescriptive targets prior to measurement of CAEPs.

The present study found that the activation of NLFC in hearing aids significantly increased aided sensation levels for $/ \mathrm{t} /$ and $/ \mathrm{s} /$. There was also a significant increase in the detection rate of CAEPs for $/ \mathrm{t} /$ and (insignificantly) for $/ \mathrm{s} /$. There was no difference in aided sensation levels of $/ \mathrm{g} /$ between the two NLFC conditions, as would be expected given that the spectral peak of energy was at a frequency region lower than the lowest cutoff frequency in the NLFC hearing aids. The detection rates of CAEPs for /g/ were close to ceiling for both NLFC conditions. The current findings suggest that the CAEPs provide information about audibility both before and after the feature is invoked. This supports the use of measurements of aided CAEPs for validating hearing aid fitting. Speech stimuli presented at suprathreshold levels that evoke a neural response at the auditory cortex suggests that they are likely to be perceived behaviorally. The relationship between the presence of cortical responses and the children's real-world functional performance will be examined in future research. As this study focused on the presence or absence of CAEPs for hearing aid evaluation, future work will also examine whether the morphology of neural responses evoked by different speech sounds would shed light on the discriminability of sounds with amplification.

\section{CLINICAL IMPLICATIONS}

To facilitate clinical applications of measuring CAEPs for validation of amplification, the likelihood of presence of CAEPs for $/ \mathrm{t} /$ and /g/ when CAEPs for /s/ was present was examined by cross-tabulation (see Fig. 5).

Of the 148 recordings of CAEPs using /s/ as stimulus, the detection rate was $69 \%$. Findings indicate that when CAEPs were present for $/ \mathrm{s} /$, cortical responses for $/ \mathrm{t} /$ were detected for 


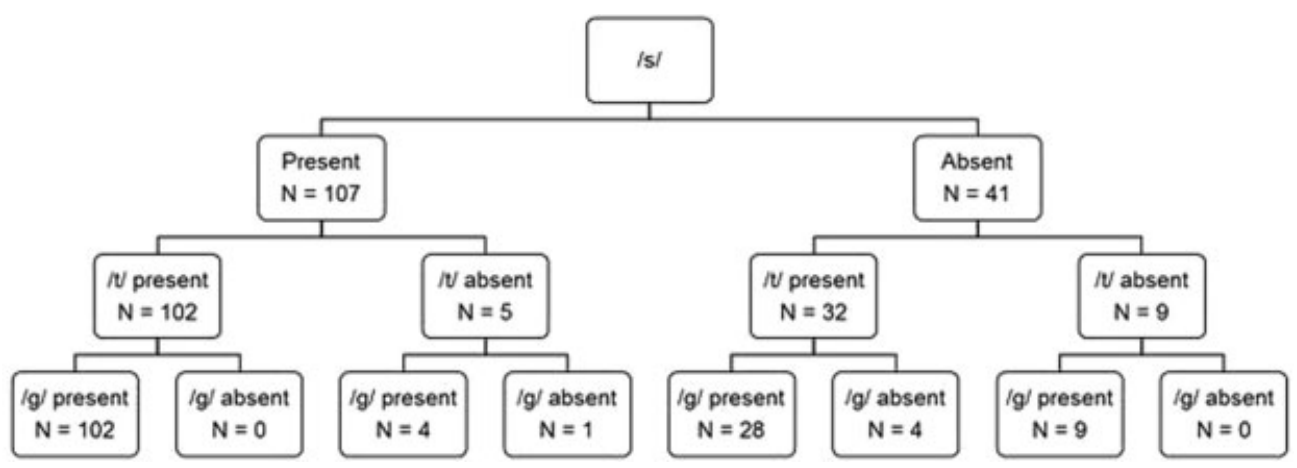

Figure 5 Cross-tabulation of results of cortical auditory evoked potentials for $/ \mathrm{g} / \mathrm{l} / \mathrm{t} /$, and $/ \mathrm{s} /$.

95\% (102 of 107 recordings), of which CAEPs for $/ \mathrm{g} /$ were present $100 \%$ of the time (chi-square $=20.6, \mathrm{df}=1, p<0.0001)$. For the 41 measures of $/ \mathrm{s} /$ when CAEPs were absent, cortical responses for $/ \mathrm{t} /$ were detected for $78 \%$ ( 32 of 41 recordings), of which $88 \%$ ( 28 of 32 recordings) had CAEPs for $/ \mathrm{g} /$ (chisquare $=1.25, \mathrm{df}=1, p=0.2$ ). Of the 9 measures of / $t /$ when CAEPs were absent, all had CAEPs for $/ \mathrm{g} /$.

After verifying that hearing aids matched prescriptive targets, validation of the fit using speech-evoked CAEPs for an individual may proceed with a clinical protocol that commences testing with the stimulus $/ \mathrm{s} /$. No further assessments are warranted if cortical responses for $/ \mathrm{s} /$ are detected. In line with the Australian Hearing protocol for CAEP testing (see Punch et $\mathrm{al}$, this issue), ${ }^{35}$ the stimulus /s/ can be presented first at $65 \mathrm{~dB}$ SPL, then at $55 \mathrm{~dB}$ SPL if CAEPs were present at $65 \mathrm{~dB}$, or at 75 $\mathrm{dB}$ SPL if CAEPs were absent. The results can be used to guide hearing aid adjustment (see Punch et $\mathrm{al}^{35}$ ). If CAEPs were absent at $75 \mathrm{~dB}$ SPL, NLFC may be implemented to increase audibility and verified by measurements of CAEPs. On the other hand, if CAEPs for $/ \mathrm{s} /$ were absent despite optimized amplification, testing may proceed with $/ \mathrm{t} /$ as stimulus to assess audibility at lower frequencies so that hearing aids may be adjusted to increase access to speech sounds. If validation of fitting at lower frequencies is desired, testing may proceed using $/ \mathrm{g} /$ as stimulus. If there is concern that audibility of very low frequencies may be compromised by using a closed earmold, testing may proceed with using $/ \mathrm{m} /$ as stimulus. Compared with the established protocol of measuring CAEPs with three stimuli $(/ \mathrm{m} /, / \mathrm{t} /, / \mathrm{g} /)$ at two levels (see Punch et al, this issue), ${ }^{35}$ this proposed approach will reduce clinical test time (one or two stimuli at two levels instead of three stimuli at two or more levels) and will also increase knowledge about a child's access to high frequencies to guide clinical management.

For assessing effectiveness of signal-processing technology that aims to increase audibility, the measurement of CAEPs (or other objective methods) may be an effective method for quantifying the variation in audibility due to the technology. It will be necessary to complement measurement of CAEPs with speech production or perception measures, as findings in previous studies that evaluated NLFC technology for school-aged children suggested that NLFC may increase audibility of /s/ and / $t /$ that is otherwise not possible with conventional processing but may compromise the discriminability of other sounds (for reviews, see Ching $^{36}$ and McCreery et $\mathrm{al}^{37}$ ).

In some children, it may not be possible to evoke a cortical response with any degree of amplification. The proportion is higher in younger than in older children. As current knowledge in regards to the practical implications of absent cortical responses for spoken language development of children is limited, continual monitoring of developmental outcomes will be necessary (e.g., Golding et $\left.\mathrm{al}^{20}\right)$. For children with severe to profound hearing loss at the most impaired frequencies, it may not be possible to provide amplification sufficient to evoke a cortical response to conversational level speech sounds. For example, a 
child with absent cortical responses despite optimized hearing aid fitting who also presents with delays in aided functional performance indicates the need to consider cochlear implant candidacy evaluation and/or the use of alternative modes of communication. Expediting decisions to implant early will enable the child to reap the benefits of early identification and intervention for supporting spoken language development. $^{38}$

\section{CONCLUSIONS}

In this study, aided CAEPs evoked by /g/, /t/, and /s/ from a sample of children with mild to severe hearing loss were evaluated. Results indicate that aided cortical responses to speech stimuli at positive sensation levels were present for 93,90 , and $76 \%$ for $/ \mathrm{g} / \mathrm{h} / \mathrm{t} /$, and $/ \mathrm{s} /$, respectively. On average, activation of NLFC increased aided sensation levels for $/ \mathrm{t} /$ and $/ \mathrm{s} /$. It also led to an increase in detection rates of CAEPs for $/ \mathrm{t} / \mathrm{and} / \mathrm{s} /$. The study shows that measurements of CAEPs provide information about audibility before and after NLFC was activated and lends support to using the method for hearing aid evaluation. Based on current results, a clinical protocol for validation of hearing aid fitting by measuring CAEPs with speech stimuli is proposed.

\section{ACKNOWLEDGMENTS}

This work was partly supported by National Institutes of Health/National Institute on Deafness and Other Communication Disorders (NIH NIDCD R01-DC008080). The authors also acknowledge the financial support of the Commonwealth of Australia through the Department of Health, the Office of Hearing Services, and the establishment of the HEARing $\mathrm{CRC}$ and the Cooperative Research Centres. The authors thank the families and children who participated in this study. We also thank Angela Wong for her assistance with data collection and Kirsty Gardner-Berry for her comments for assistance with presenting portions of this paper at the XXIV Biennial Symposium of the International Evoked Response Audiometry Study Group, May 10-14, 2015 in Busan, Korea. Thanks are also due to the reviewers who provided detailed comments for improvements on an earlier version of this manuscript.

\section{REFERENCES}

1. Seewald RC, Scollie SD. An approach for ensuring accuracy in pediatric hearing instrument fitting. Trends Amplif 2003;7(1):29-40

2. Bagatto MP, Moodie ST, Seewald RC, Bartlett DJ, Scollie SD. A critical review of audiological outcome measures for infants and children. Trends Amplif 2011;15(1):23-33

3. Martin B, Tremblay K, Stapells D. Principles and applications of cortical auditory evoked potentials. In: Burkard R, Eggermont J, Don M, eds. Auditory Evoked Potentials: Basic Principles and Clinical Application. Philadelphia, PA: Lippincott Williams and Wilkins; 2007:482-507

4. Hyde M. The N1 response and its applications. Audiol Neurootol 1997;2(5):281-307

5. Kurtzberg D, Hilpert PL, Kreuzer JA, Vaughan HG Jr. Differential maturation of cortical auditory evoked potentials to speech sounds in normal fullterm and very low-birthweight infants. Dev Med Child Neurol 1984;26(4):466-475

6. Pasman JW, Rotteveel JJ, de Graaf R, Maassen B, Notermans SL. Detectability of auditory evoked response components in preterm infants. Early Hum Dev 1991;26(2):129-141

7. Gilley PM, Sharma A, Dorman M, Martin K. Developmental changes in refractoriness of the cortical auditory evoked potential. Clin Neurophysiol 2005;116(3):648-657

8. Bauer PW, Sharma A, Martin K, Dorman M. Central auditory development in children with bilateral cochlear implants. Arch Otolaryngol Head Neck Surg 2006;132(10):1133-1136

9. Sharma A, Martin K, Roland P, et al. P1 latency as a biomarker for central auditory development in children with hearing impairment. J Am Acad Audiol 2005;16(8):564-573

10. Sharma A, Gilley PM, Dorman MF, Baldwin R. Deprivation-induced cortical reorganization in children with cochlear implants. Int J Audiol 2007;46(9):494-499

11. Sharma A, Cardon G, Henion K, Roland P. Cortical maturation and behavioral outcomes in children with auditory neuropathy spectrum disorder. Int J Audiol 2011;50(2):98-106

12. Gravel JS, Kurtzberg D, Stapells DR, Vaughan HG, Wallace IF. Case studies. Semin Hear 1989; 10:272-287

13. Purdy SC, Katsch R, Dillon H, Storey L, Sharma M, Agung K. Aided cortical auditory evoked potentials for hearing instrument evaluation in infants. In: Seewald RC, Bamford JM, eds. A Sound 
Foundation through early Amplification: Proceedings of the Third International Conference. Stäfa. Switzerland: Phonak AG; 2005:115-128

14. Pearce W, Golding M, Dillon H. Cortical auditory evoked potentials in the assessment of auditory neuropathy: two case studies. J Am Acad Audiol 2007;18(5):380-390

15. Sharma A, Dorman MF, Spahr AJ. A sensitive period for the development of the central auditory system in children with cochlear implants: implications for age of implantation. Ear Hear 2002; 23(6):532-539

16. Dorman MF, Sharma A, Gilley P, Martin K, Roland P. Central auditory development: evidence from CAEP measurements in children fit with cochlear implants. J Commun Disord 2007;40(4): 284-294

17. Glista D, Easwar V, Purcell D, Scollie S. A pilot study on cortical auditory evoked potentials in children: aided CAEPs reflect improved highfrequency audibility with frequency compression hearing aid technology. Int J Otolaryngol 2012; 2012:1-12

18. Van Dun B, Carter L, Dillon H. Sensitivity of cortical auditory evoked potential detection for hearing-impaired infants in response to short speech sounds. Audiol Res. 2012;2(e13):65-76

19. Gardner-Berry K, Chang H, Ching TYC, Hou S. Detection rates of cortical auditory evoked potentials at different sensation levels in infants with sensory/ neural hearing loss and auditory neuropathy spectrum disorder. Semin Hear 2016;37(1):53-61

20. Golding M, Pearce W, Seymour J, Cooper A, Ching T, Dillon $\mathrm{H}$. The relationship between obligatory cortical auditory evoked potentials (CAEPs) and functional measures in young infants. J Am Acad Audiol 2007;18(2):117-125

21. Ching TYC, Hill M. The parents' evaluation of aural/oral performance of children (PEACH) scale: normative data. J Am Acad Audiol 2007;18(3): 220-235

22. Gardner-Berry K, Purdy SC, Ching TY, Dillon H. The audiological journey and early outcomes of twelve infants with auditory neuropathy spectrum disorder from birth to two years of age. Int J Audiol 2015;54(8):524-535

23. Rance G, Cone-Wesson B, Wunderlich J, Dowell R. Speech perception and cortical event related potentials in children with auditory neuropathy. Ear Hear 2002;23(3):239-253

24. American Academy of Audiology. American Academy of Audiology Clinical Guidelines: Paediatric Amplification. 2013. Available at: www.audiology. orghttp://www.audiology.org/publications-resources/document-library/pediatric-rehabilitationhearing-aids. Date accessed: June 2013

25. Simpson A, Hersbach AA, McDermott HJ. Improvements in speech perception with an experi- mental nonlinear frequency compression hearing device. Int J Audiol 2005;44(5):281-292

26. Ling D. Speech and the Hearing-Impaired Child: Theory and Practice. Washington, DC: Alexander Graham Bell Association for the Deaf; 1976

27. Ling D. Speech and the Hearing-Impaired Child: Theory and Practice. 2nd ed. Washington, DC: Alexander Graham Bell Association for the Deaf; 2002

28. Moeller MP, McCleary E, Putman C, Tyler-Krings A, Hoover B, Stelmachowicz P. Longitudinal development of phonology and morphology in children with late-identified mild-moderate sensorineural hearing loss. Ear Hear 2010;31(5):625-635

29. Zhang VW, Ching TYC, Van Buynder P, et al. Aided cortical response, speech intelligibility, consonant perception and functional performance of young children using conventional amplification or nonlinear frequency compression. Int J Pediatr Otorhinolaryngol 2014;78(10):1692-1700

30. King AM. The national protocol for paediatric amplification in Australia. Int J Audiol 2010;49 (Suppl 1):S64-S69

31. Byrne D, Dillon H, Ching T, Katsch R, Keidser G. NAL-NL1 procedure for fitting nonlinear hearing aids: characteristics and comparisons with other procedures. J Am Acad Audiol 2001;12(1):37-51

32. Ching TYC, Dillon H. Prescribing amplification for children: adult-equivalent hearing loss, real-ear aided gain, and NAL-NL1. Trends Amplif 2003; 7(1):1-9

33. Bagatto MP, Scollie SD, Seewald RC, Moodie KS, Hoover BM. Real-ear-to-coupler difference predictions as a function of age for two coupling procedures. J Am Acad Audiol 2002;13(8):407-415

34. Chang HW, Dillon H, Carter L, van Dun B, Young ST. The relationship between cortical auditory evoked potential (CAEP) detection and estimated audibility in infants with sensorineural hearing loss. Int J Audiol 2012;51(9):663-670

35. Punch S, Van Dun B, King A, Carter L, Pearce W. Clinical experience of using cortical auditory evoked potentials in the treatment of infant hearing loss in Australia. Semin Hear 2016;37(1):36-52

36. Ching TYC. Hearing aids for children. In: Wong L, Hickson L, eds. Evidence-Based Practice in Audiology: Evaluating Interventions for Children and Adults with Hearing Impairment. San Diego, CA: Plural Publishing; 2012:93-118

37. McCreery RW, Venediktov RA, Coleman JJ, Leech HM. An evidence-based systematic review of frequency lowering in hearing aids for school-age children with hearing loss. Am J Audiol 2012; 21(2):313-328

38. Ching TYC, Day J, Cupples L. Phonological awareness and early reading skills in children with cochlear implants. Cochlear Implants Int 2014;15(Suppl 1):S27-S29 